\title{
Functional and evolutionary implications of a survey of various actinomycetes for homologues of two Streptomyces coelicolor sporulation genes
}

\author{
J. Soliveri, $\dagger$ E. Vijgenboom, $\ddagger$ C. Granozzi,§ K. A. Plaskitt and K. F. Chater* \\ John Innes Institute, John Innes Centre, Colney Lane, Norwich NR4 7UH, UK
}

(Received 16 June 1993; accepted 22 July 1993)

\begin{abstract}
In Streptomyces coelicolor $\mathrm{A3}(2)$ the whiB and whiG genes are essential for sporulation, their deduced products being a possible transcriptional activator and an RNA polymerase sigma factor, respectively. In a survey of DNA from diverse actinomycetes by Southern blotting, all samples tested hybridized with whiB, but only those representing genera capable of producing sporulating aerial mycelium hybridized with whiG. It is postulated that whiB may play a more intimate role in hyphal fragmentation processes (including sporulation) than whiG. The $w h i B$ and whiG homologues (whiB-Stv and whiG-Stv) of Streptoverticillium griseocarneum were cloned and sequenced, and subjected to functional tests in $S$. coelicolor whiB and whiG mutants. The genes were closely similar, but not identical, to their $S$. coelicolor counterparts at the DNA and deduced protein levels, and both Stv. griseocarnum gene products could function well in $S$. coelicolor. However, studies with hybrid transcription units suggested that the promoter region of whiB-Stv is somewhat inefficient in $S$. coelicolor.
\end{abstract}

\section{Introduction}

Actinomycetes are Gram-positive prokaryotes with DNA rich in $\mathrm{G}+\mathrm{C}$. Many of them exhibit some degree of filamentous growth, with only distantly spaced crosswalls, alternating with fragmentation into cells containing single genomes (Goodfellow \& Cross, 1983). In some genera the filamentous habit persists throughout vegetative growth, and quasi-exponential growth is achieved by branching, giving a mycelium. Such mycelial

${ }^{*}$ Author for correspondence. Tel. +4460352571 ; fax +44603 56844 .

† Present address: Departamento de Microbiología y Parasitología, Universidad de Alcalá de Henares, 28871-Alcalá de Henares, Madrid, Spain.

$\ddagger$ Present address: Gorlaeus Laboratoria, ASKAM, PO Box 9502, 2300 RA Leiden, The Netherlands.

§ Present address: Lepetit Stabilimento di Brindisi, Casella Postale 199, 72100 Brindisi, Italy.

The nucleotide sequence data reported in this paper have been submitted to the EMBL Data Library and have been assigned the accession numbers X68708 (for whiB-Stv) and X68709 (for whiG-Stv). organisms often produce morphologically specialized structures, such as sporangia in Actinoplanes or aerial hyphae in Streptomyces, where large numbers of uninucleoidal spores can be formed which allow dispersal to take place. This developmental complexity has been most studied in Streptomyces coelicolor A3(2) (see Chater, 1989, for a recent general review). One focus of attention has been on genes which are needed for the formation of the specialized sporulation septa that divide aerial hyphal tips into spore compartments (Wildermuth \& Hopwood, 1970), but are dispensable for normal growth. These early sporulation genes were identified by morphological studies (Chater, 1972; McVittie, 1974) of a collection of mutants unable to form the normal grey spore pigment (Hopwood et al., 1970). Because their aerial mycelium remained white on prolonged incubation, these mutants were designated whi mutants. Genetic analysis (Chater, 1972) revealed at least six loci (whiA, B, C, G, $H$ and $I$ ) at which mutations caused deficiencies in sporulation septation. Among these, whiA, $B, G$ and $H$ mutations seemed to cause a complete absence of sporulation septa (Chater, 1972; McVittie, 1974) (Davis \& Chater, 1992; Mendez \& Chater, 1987; Chater et al., 1989). Two of the corresponding genes, whiB and whiG, have been cloned and sequenced. The whiB gene product, WhiB, is deduced to contain only 87 
amino acids, including four cysteine and two tryptophan residues as well as an unusually high content of charged residues (acidic at the amino terminus and mostly basic at the carboxy terminus) (Davis \& Chater, 1992). No obvious similarities of WhiB to other known or deduced proteins were discovered, though a number of transcription-factor-like aspects of WhiB were pointed out (Davis \& Chater, 1992). The deduced whiG gene product, however, closely resembles the RNA polymerase sigma factors $\sigma^{\mathrm{D}}$ of Bacillus subtilis and $\sigma^{\mathrm{F}}$ of enteric bacteria (Chater et al., 1989; Helmann, 1991; Starnbach \& Lory, 1992), and is here termed $\sigma^{\text {WhiG }}$, though there is no direct evidence of its mode of action. It is required (presumably directly) for transcription of promoters with sequence features resembling the consensus features of $\sigma^{\mathrm{D}}$ - and $\sigma^{\mathrm{F}}$ dependent promoters (Tan \& Chater, 1993). Additional copies of whiG cause excessive and ectopic sporulation, implying that $\sigma^{\text {WhiG }}$ crucially controls a developmental switch (Chater et al., 1989).

Both whiB- and whiG-related sequences were detectable by Southern blotting in more than 20 Streptomyces spp. tested (Chater et al., 1989; Blanco et al., 1993), bearing out the reasonable expectation that sporulation in all Streptomyces spp. would involve some common elements. It is less easy to predict whether homologous genes are required for sporulation or fragmentation processes in other actinomycete genera: information that may be relevant to understanding the functions of the genes and the evolution of differentiation in this morphologically diverse order. To help in addressing these questions, we here describe the use of $w h i B$ and whiG sequences as probes in Southern blots against various actinomycetes and other bacteria, leading to the cloning, sequencing and functional testing of homologues from a Streptoverticillium species.

\section{Methods}

Bacterial strains, plasmids and transformation. The cultivation and manipulative procedures were as in Hopwood et al. (1985) and Sambrook et al. (1989). Streptomyces coelicolor A3(2) derivatives were: $\mathrm{C} 70$ (whiB 70 ) and $\mathrm{C} 71$ (whiG7l), both derived from the wild-type after mutagenesis (Chater, 1972); J171 (whiB218 cysD18 straAl NF; Chater, 1972) and J1501 [hisAl uraA1 strAl $\mathrm{Pgl}^{-}\left(\equiv \phi \mathrm{C}^{-} 1^{\mathrm{S}}\right) \mathrm{SCP}^{-} \mathrm{SCP2}^{-}$; Chater et al., 1982]. Streptomyces lividans 66 (John Innes Institute strain 1326) was the host for some transformation experiments. The source of DNA for cloning of whiB and whiG homologues was Streptoverticillium griseocarneum NCIMB 40447. Actinomycetes were maintained on R2YE agar (Hopwood et al., 1985). Morphological studies were done on suitably supplemented MM containing $0.5 \%$ mannitol as carbon source (Hopwood et al., 1985). pIJ558 (provided by Dr N. K. Davis), containing an $819 \mathrm{bp} B c I$ - $B s t$ EII fragment including whiB inserted into pUC19 (Yanisch-Perron et al., 1985), was the source of whiB probe DNA. pIJ486 (Ward et al., 1986) was used as a general vector for Streptomyces. Escherichia coli JM101 was the host for M13 mp18, M13 mp19 and pUC19 derivatives (Norrander et al., 1983). Transformation conditions for Streptomyces were as in
Hopwood et al. (1985), and for E. coli they were as in Sambrook et al. (1989). Eight plasmids containing permutations of whiBP1, whiBP2 and the whiB ORF from $S$. coelicolor and the equivalent regions of whiB-St $v$ were constructed from the following modules (coordinates from Fig. 7 and Davis \& Chater, 1992): S. coelicolor P1, 181 bp $B c I-B g I I$ fragment; $S t v$. ' $\mathrm{P} 1$ ', ca. $500 \mathrm{bp}$ fragment extending from the upstream end of the sequence to the BgIII site; $S$. coelicolor P2, $116 \mathrm{bp}$ $B g l I I-N d e$ I fragment from a mutagenized whiB sequence in which the CACATG hexamer including the whiB start codon had been modified to CATATG (NdeI site) by oligonucleotide-directed mutagenesis (Kunkel et al., 1987); Stv. 'P2', 116 bp BglII-NdeI fragment from a similarly mutagenized whiB-Stv gene; $S$. coelicolor whiB ORF, 531 bp $N d e I-B s t$ EII fragment from the modified ( $N$ deI site-containing) whiB clone; whiB-Stv ORF, 293 bp NdeI-XmaIII fragment from the similarly modified whiB-St $v$ clone. The constructions were assembled in pIJ2181, a pUC19 derivative from which the $N d e I$ site had been removed by filling-in the ends of pUC19 linearized by NdeI. The completed constructions were inserted into pIJ486, using the HindIII and EcoRI sites, and they were all oriented anticlockwise in pIJ486 so that they were preceded by a transcription terminator present in the vector (Ward et al., 1986)

Sources of total DNA. DNA was also isolated from the following Streptoverticillium species: Stv. griseocarneum NCIMB 40447; Stv. albireticuli ATTC 19721; Stv. baldacci ATCC 23654; Stv. distallicum DSM 40846; Stv. hachijoense ATCC 19769; Stv. ladakanum ATCC 27441; Stv. orinoci ATCC 23202; and Stv. rectiverticillatum ATCC 25491, as well as from Streptosporangium viridogriseum subsp. viridogriseum ATCC 25242 and Actinoplanes brasiliensis (Lepetit). The following strains were donated by H.M. Kieser: Amycolatopsis mediterranea ATCC 13685; Arthrobacter simplex NCIB 9770; Bacillus subtilis 168; Corynebacterium fascians (= Rhodococcus fascians) JII 2767; Micromonospora chalcea var. florida (J. E. Davies); Nocardia corallina; and Saccharopolyspora erythraea NRRL 2338. R. Melton provided DNA from Mycobacterium tuberculosis var. bovinus BCG. D. A. Hodgson provided DNA from Myxococcus xanthus. J.A. Downie provided DNA of Rhizobium leguminosarum 8400 and its derivative lacking the Sym plasmid.

Techniques for DNA manipulation in vitro. Conventional procedures were used for nuclease digestion, ligation, Southern blots, plaque lifts, DNA preparation, random priming (Amersham, oligolabelling kit) and polymerase chain reaction (Hopwood et al., 1985; Sambrook et al., 1989). Hybond-N membranes (Amersham) were used for Southern blotting, and cellulose nitrate membranes (Sartorius) for plaque hybridization. Hybridization conditions were as in Hopwood et al. (1985), with the most stringent wash being done at $65^{\circ} \mathrm{C}$ in $0.1 \%$ SDS, $3 \times$ SSC.

DNA sequencing. The dideoxy chain-terminating method (Sanger $e t$ al., 1977) was used on single-stranded DNA templates of exonucleaseIII-generated clones (Henikoff, 1986) in M13 mp18 or mp19, using the Sequenase kit (US Biochemical Corp.) with universal primer or oligonucleotide primers based on earlier rounds of sequencing. Open reading frames were identified by the program FRAME (Bibb et al., 1984). Further processing and analysis of the sequences were by the Sequence Analysis Software Package of the University of Wisconsin Genetics Computer Group (Devereux et al., 1984).

Microscopy. For phase-contrast microscopy, samples were prepared by the cover-slip method (Chater, 1972). Electron microscopy of uranyl-acetate-stained thin sections was as in Chater et al. (1989). Scanning electron microscopy (model SX-25, I.S.I.) was done on goldand palladium-coated cultures (Cross et al., 1973) grown on Bennett agar against cover slips inserted into the agar at an angle (Locci $e t$ al., 1969). 


\section{Results}

$D N A$ hybridizing with the whiB gene of $S$. coelicolor is present in representatives of many actinomycete genera

DNA samples from representatives of nine actinomycete genera other than Streptomyces (Fig. 1) were digested with $P v u I I$ and subjected to Southern blotting. The filters were probed, using hybridization conditions of intermediate stringency, with a ${ }^{32} \mathrm{P}$-labelled whiB probe consisting of a $467 \mathrm{bp} \mathrm{ApaI}$ fragment that contained the 264 bp whiB ORF and some flanking non-coding DNA (extending $122 \mathrm{bp}$ upstream and $81 \mathrm{bp}$ downstream of whiB). With one exception, a single perceptible signal was obtained with every DNA sample (Fig. 2a), in the following order of decreasing signal strength: Streptoverticillium and Arthrobacter, Amycolatopsis, Mycobacterium, Corynebacterium, Nocardia, Actinoplanes, Micromonospora and Saccharopolyspora. We have not tested whether the two bands obtained with Arthrobacter DNA reflected two whiB homologues, or the presence of an internal $P v u I I$ site, or incomplete digestion. In another experiment (not shown) seven other Streptoverticillium spp. gave signals of similar intensity to that shown in Fig. 1 for Stv. griseocarneum DNA, though to PvuII

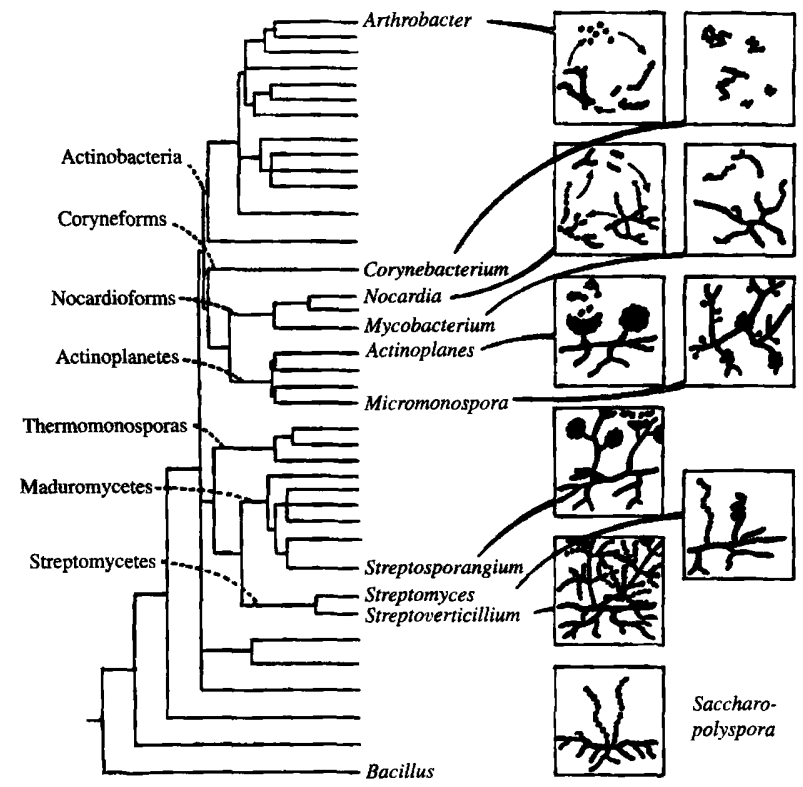

Fig. 1. Actinomycete genera analysed by Southern blotting: their phylogenetic positions and morphology. The phylogenetic tree is based on partial sequencing of $16 \mathrm{~S}$ rRNA and is redrawn from Goodfellow (1989). The vignettes of morphology are redrawn from Goodfellow \& Cross (1983). To simplify the diagram, only those genera mentioned in the text are named. The molecular phylogenetic position of Saccharopolyspora erythraea and Amycolatopsis mediterranea have not been established. Both were formerly designated as streptomycetes, but more recent conventional taxonomy (Lechevalier, 1989) has grouped both with nocardioforms. A. mediterranea resembles Nocardia spp. in its morphology. fragments mostly of different sizes; and a weaker signal was also obtained with a Streptosporangium sp. Overall, these results suggested the universal occurrence of whiB homologues among actinomycetes, raising the question of whether they might also occur in other, phylogenetically distant, bacterial genera. In a limited survey, the $w h i B$ probe was used to analyse DNA of Bacillus subtilis, which is Gram-positive and has about $45 \%$ $\mathrm{G}+\mathrm{C}$ in its DNA, and three Gram-negative species: Escherichia coli (ca. $50 \% \mathrm{G}+\mathrm{C}$ ), Myxococcus xanthus (ca. $70 \% \mathrm{G}+\mathrm{C}$ ) and Rhizobium leguminosarum (ca. 60\% $\mathrm{G}+\mathrm{C}$, containing the $\mathrm{SYM}$ plasmid $\mathrm{pRL} 1$ which encodes many-but not all - of the functions needed for the Rhizobium-legume symbiosis: Downie \& Surin, 1990). A clear signal was obtained with $R$. leguminosarum DNA, but not with the other samples (Fig. 3). The signal was unchanged in DNA from strain 8400 lacking pRL1. A more detailed study than is currently possible would be necessary to evaluate further whether relatively diverged whiB homologues are present in B. subtilis, E. coli or $M$. xanthus.

Close homologues of the whiG gene of $S$. coelicolor are absent from some actinomycetes

The same set of actinomycete DNA samples was analysed for the presence of DNA hybridizing with whiG, using a probe obtained by PCR amplification of the part of the whiG sequence encoding much of region 2 of $\sigma^{\text {WhiG }}$ (nt 288-529, coordinates of Chater et al., 1989) - a region particularly similar at the amino acid level to $\sigma^{\mathrm{D}}$ of B. subtilis (Helmann et al., 1988) and $\sigma^{\mathrm{F}}$ of Salmonella typhimurium (Ohnishi et al., 1990), and therefore likely to be particularly highly conserved even at the DNA level among diverse actinomycetes, with their high $\mathrm{G}+\mathrm{C}$ content and phylogenetic relatedness. Surprisingly, and in contrast to the results for whiB, homologous sequences were detected only in the Saccharopolyspora, Streptoverticillium (including seven spp. that are not shown) and Amycolatopsis spp., and were absent from the Arthrobacter, Corynebacterium, Nocardia, Mycobacterium, Actinoplanes and Micromonospora spp. (Fig. $2 \mathrm{~b}$ ). The use of a presumptively conserved region of whiG as a probe, rather than the whole whiG gene, and of relatively non-stringent hybridization conditions, probably explains why we were readily able to detect a signal in Amycolatopsis mediterranea, which had previously appeared to lack a whiG homologue (Chater et al., 1989).

Using the same probe, a putative whiG homologue was detected in DNA of $E$. coli JM101, presumably corresponding to the as yet unsequenced $E$. coli gene specifying $\sigma^{\mathrm{F}}$, but none was detected in DNA of $B$. subtilis, despite the presumed presence of the sigD gene 


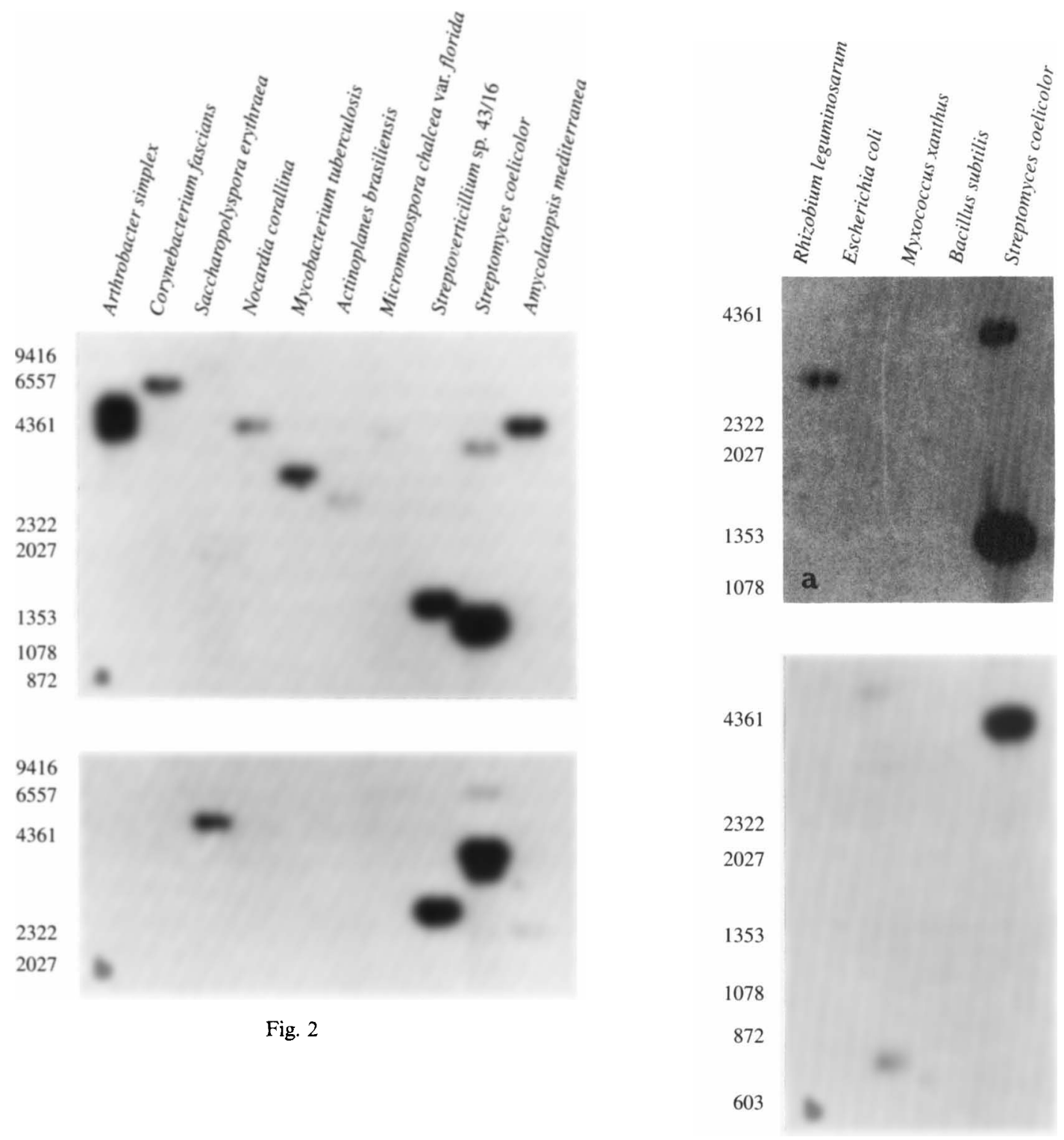

Fig. 3

Fig. 2. Southern blot analysis of DNA from various actinomycetes, using (a) whiB-and (b) whiG-specific probes. DNA samples were digested with $P v u I I$. The most stringent wash after hybridization was with $3 \times \mathrm{SSC}$ at $65^{\circ} \mathrm{C}$. See Results for details of probes, and Methods for details of blotting and hybridization. Streptoverticillium sp. 43/16 is synonymous with Str. griseocarneum.

Fig. 3. Southern blot analysis of DNA from unicellular bacteria, using (a) whiB- and (b) whiG-specific probes. The probes and conditions were as in Fig. 2. DNA samples were digested with PvuII.

encoding $\sigma^{\mathrm{D}}$. Examination of the $B$. subtilis sigD DNA sequence (Helmann et al., 1988) reveals that even the most conserved parts of the region corresponding to the probe do not have more than $50 \%$ sequence identity to whiG over extended segments, whereas the Salmonella typhimurium fliA gene, encoding $\sigma^{\mathrm{F}}$ (Ohnishi et al., 1990) and presumably very similar to its $E$. coli homologue, has segments of several tens of nucleotides with $60-70 \%$ identity to the whiG probe. It is not known whether Rhizobium leguminosarum or Myxococcus xanthus, which did not give signals, contain a comparable gene. Nevertheless, the apparent success in detecting a signal in an organism as remote from actinomycetes in phylogeny and DNA base composition as $E$. coli gives us confidence that the absence of a signal from many actinomycetes indicates the absence of a whiG-like gene. 


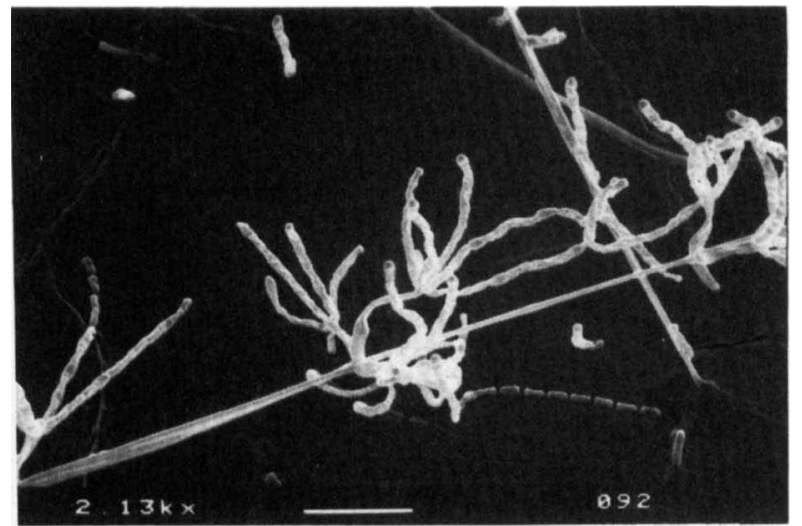

Fig. 4. Scanning electron micrograph of Stv. griseocarneum. Bar marker, $5 \mu \mathrm{m}$. Note the occurrence of multiple whorls of branches from the principal aerial hypha. Each branch itself forms a multiple branch point from which spore-forming hyphae develop.
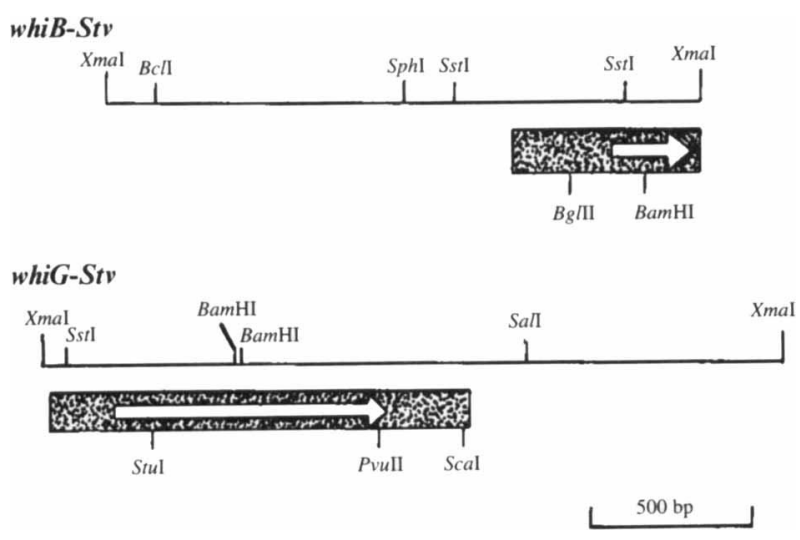

Fig. 5. Restriction maps of cloned Stv. griseocarneum DNA that hybridized with whiB-and whiG-specific probes. The regions sequenced are shaded, and the positions and orientations of the whiB-St $v$ and whiG-Stv ORFs are shown by arrows.

\section{Cloning from Streptoverticillium griseocarneum of DNA} hybridizing with $S$. coelicolor whiB and whiG probes

To assist in understanding the significant features of $w h i B$ and $w h i G$, the homologue of each was cloned from Streptoverticillium griseocarneum. The genus Streptoverticillium is closely related to Streptomyces (Goodfellow, 1989), but Streptoverticillium shows a distinctive morphology during aerial mycelium development (Fig. 4): it forms whorls of branches before sporulation, in contrast to the simple lateral branching (if any) of Streptomyces aerial hyphae (Locci, 1989). This difference in aerial mycelium branching pattern might conceivably be reflected - or caused - by differences in sporulation regulatory genes such as whiG or whiB. DNA of Stv. griseocarneum was subjected to Southern blotting analysis, to identify suitable fragments for cloning. As a result, $X m a I$ fragments of ca. $1.9 \mathrm{~kb}$ ( $w h i B$-homologous) and ca. $2.4 \mathrm{~kb}$ (whiG-homologous) were isolated from agarose gels and inserted into the $X m a I$ site of M13 mp18. The desired clones, presumptively carrying whiB-Stv (pIJ2174) and whiG-Stv (pIJ2177), were identified by plaque hybridization. The restriction maps of representative examples are shown in Fig. 5.

The cloned Streptoverticillium DNA fragments partially restore sporulation to the appropriate $S$. coelicolor mutants

The two cloned segments of Streptoverticillium DNA were subcloned, as HindIII-EcoRI fragments, between the cognate sites of pIJ486, a multicopy vector for Streptomyces spp. The resulting plasmids (pIJ2176, containing whiB-Stv, and pIJ2179, containing whiG-Stv) were introduced into $S$. coelicolor strains $\mathbf{J} 1501$ (morphologically wild-type), C70 (a whiB70 mutant) and C71 (a whiG71 mutant). Microscopic analysis showed that C70 was induced to sporulate by $\mathrm{pIJ} 2176$, and $\mathrm{C} 71$ by pIJ2179. In neither case was complementation complete, as shown by the relatively low numbers of spores and the failure of white aerial mycelium of the colonies to develop the grey colour typical of abundantly sporulating wild-type colonies (though they were slightly greyer than the whi mutants carrying only the vector, pIJ486).

The presence of multiple copies of whiB-Stv (on pIJ2176) in the sporulation-proficient strain J1501 had no marked effect on colony morphology or sporulation. However, multiple copies of whiG-Stv (pIJ2179) caused J1501 to grow as small, black colonies with little obvious aerial mycelium, and electron microscopy (Fig. 6) revealed that the substrate mycelium of such colonies contained numerous spores - a feature previously shown to result from the presence of multiple copies of the $S$. coelicolor whiG gene (Chater et al., 1989).

These experiments indicated that the two Streptoverticillium genes were probably structurally intact and functionally closely similar to their $S$. coelicolor homologues, but also suggested that neither gene was fully efficient in the heterologous host.

The sequence of whiB-Stv is highly similar to that of the $S$. coelicolor whiB gene

To localize whiB-Stv in the $1.9 \mathrm{~kb}$ fragment cloned, a nested set of exonuclease-III-generated unidirectional deletions of the M13 mp18 clone pIJ2174 was generated from the end of the insert closer to the universal sequencing primer site. Samples taken at different times during exonuclease III treatment were analysed by Southern blotting, using the $S$. coelicolor whiB probe. The hybridization signal was not detectable when more than $0.45 \mathrm{~kb}$ had been deleted, showing that the region hybridizing to the probe was close to the universal sequencing primer site. Computer analysis of the nucleotide sequence of this region ( $593 \mathrm{bp}$ ) revealed a whiB-like 


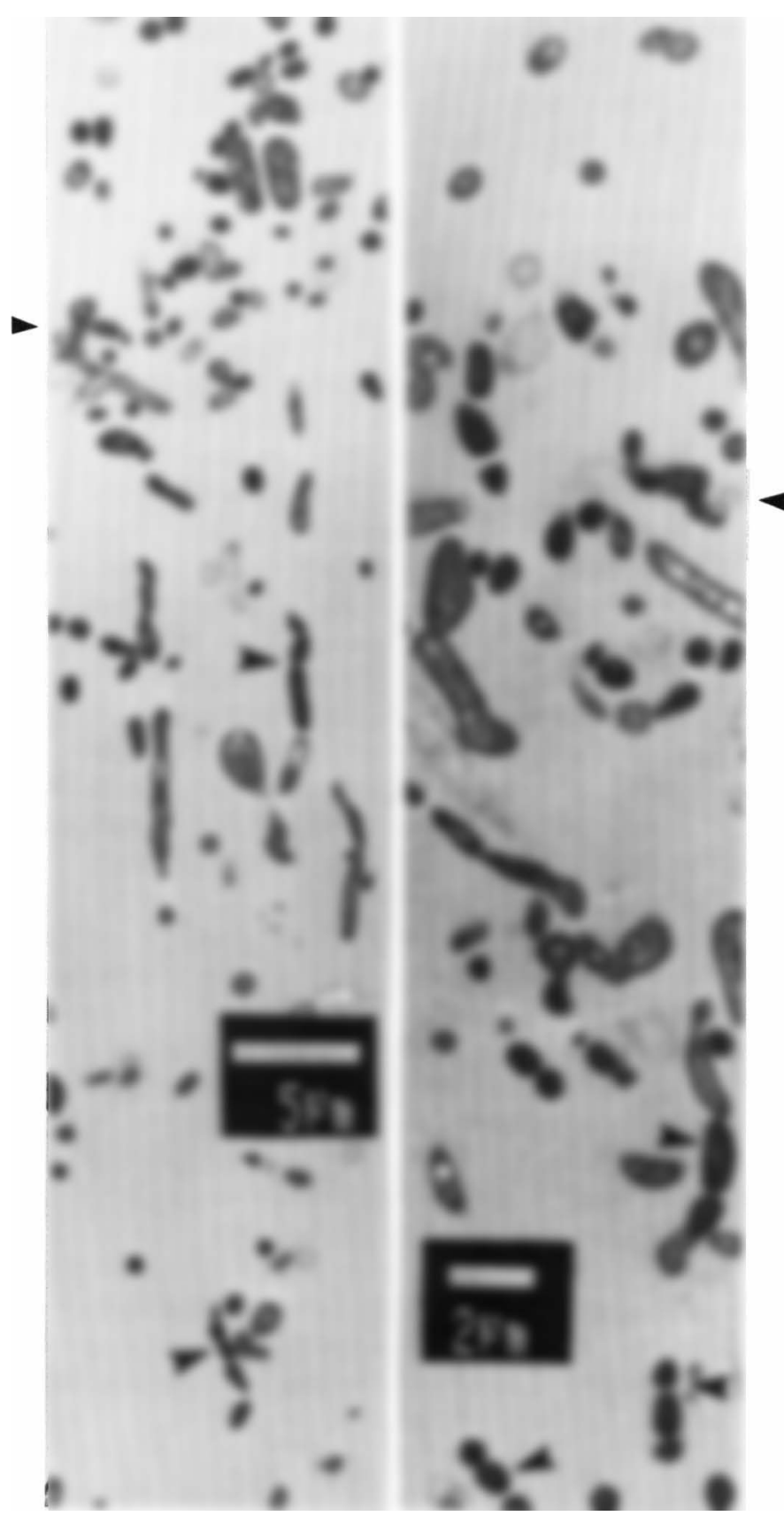

Fig. 6. Electron micrographs of a thin section of a colony of $S$. coelicolor containing multiple copies of whiG-Stv. Strain J1501/pIJ2179 was grown on $\mathrm{MM}$ containing mannitol $(0.5 \%)$ and thiostrepton $\left(5 \mu \mathrm{g} \mathrm{ml}^{-1}\right)$. A colony was examined after $4 \mathrm{~d}$. Large arrowheads show spore chains, and small arrowheads show the position of the agar surface.

ORF ( $w h i B-S t v$ ) preceded by a weak ribosome-binding site and $303 \mathrm{bp}$ of upstream sequence (Fig. 7). An alternative translation start at a GTG codon at positions $238-240$ is less likely because it is not preceded by a ribosome-binding site, it would invoke the use of several rarely used codons, and it would differ significantly from the $S$. coelicolor homologue (which has a TAA stop codon corresponding to positions $253-255$ in Fig. 7). Like WhiB protein from $S$. coelicolor, WhiB-Stv contains 87 amino acids. There are differences between the two proteins at only three positions, and each difference is conservative. At the nucleotide level, the two ORFs are $88 \%$ identical. In the non-coding 150 bp immediately upstream of the ORFs there is also extensive sequence identity: this region includes the developmentally regulated promoter whiBP2 identified by Soliveri et al. (1992) (Fig. 7). Upstream of -150 the sequences diverge, although about $55 \mathrm{nt}$ can be identified with punctuated similarity to the constitutive low level promoter whiBP1 identified in S. coelicolor (Soliveri et al., 1992; Fig. 7).

Experiments with hybrid whiB transcription units show that the product of whiB-Stv is fully functional in $S$. coelicolor

In view of the lack of knowledge about the molecular function of WhiB protein, we wished to establish whether the three amino acid differences between WhiB-Stv and WhiB of $S$. coelicolor were responsible for the apparently suboptimal complementation of the whiB70 mutant by $w h i B-S t v$. This was addressed by constructing a series of six hybrid transcription units, using modules that contained the whiBP1, whiBP 2 and whiB-coding regions of $S$. coelicolor and the equivalent segments of $S t v$. griseocarneum. These constructions exploited common $B g l \mathrm{II}$ sites separating $\mathrm{P} 1$ from $\mathrm{P} 2$, and $N d e \mathrm{I}$ sites introduced at the start of each coding region by sitedirected mutagenesis. The Stv. griseocarneum and $S$. coelicolor whiB regions were also reconstructed from the appropriate modules. The constructs were introduced into two different $S$. coelicolor whiB mutants, C70 (whiB70 point mutant) and $\mathrm{J} 171$ (whiB218 deletion). There were four main observations.

(i) All the constructs gave some degree of complementation, but this was much more obvious in the J171 deletion mutant, which was caused in every case to form sporulating grey aerial mycelium. In the $\mathrm{C} 70$ point mutant, complementation even by the reconstructed $S$. coelicolor whiB was manifested as a faint greyness in the aerial mycelium, and phase-contrast microscopy of impression preparations revealed mainly long, helical, non-sporulating aerial hyphae together with a few small areas containing spore chains (Fig. 8). These observations suggest that whiB70 is partially dominant over the wild-type allele.

(ii) In $\mathrm{J} 171$, the reconstructed $S$. coelicolor whiB gene (i.e. with its own $\mathrm{P} 1+\mathrm{P} 2$ region) gave rapid sporulation, whereas the $S$. coelicolor whiB ORF downstream of a promoter region that included either 'P1' or 'P2', or both, from Stv. griseocarneum gave full sporulation only after a further day. Thus, there is a difference in the 
1

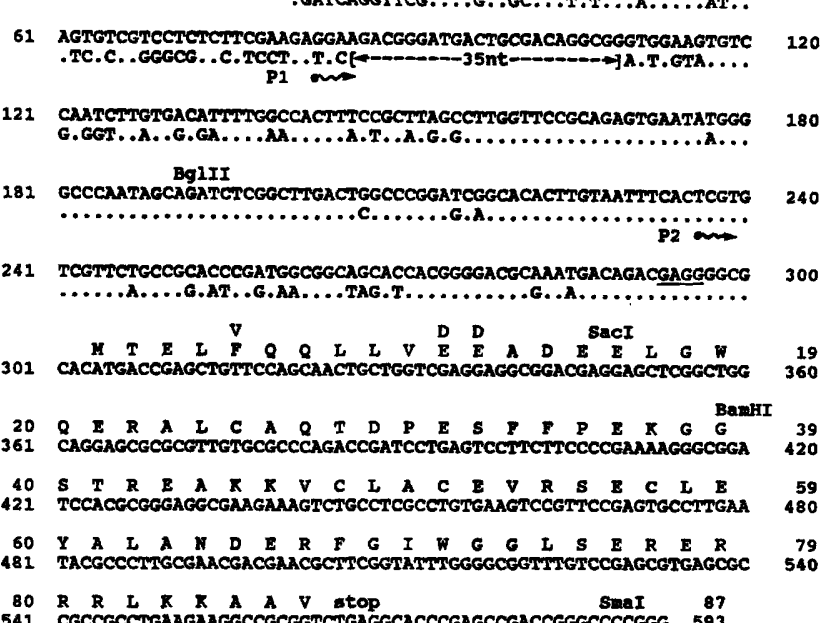

Fig. 7. DNA sequence of whiB-Stv. A purine-rich potential ribosomebinding site is underlined. Dots under the sequence mark base positions at which the sequenced region upstream of the $S$. coelicolor whiB gene (Davis \& Chater, 1992) is identical to that of whiB-Stv, base symbols indicating differences. Between nt positions 85 and 111, alignments were not obvious. The dots on wavy arrows show the positions of transcription start sites for the $S$. coelicolor whiB promoter region (Soliveri et al., 1992). The three positions (F5V, E11D, E12D) at which the deduced polypeptide specified by whiB-Stv differs from those of $S$. coelicolor and $S$. lividans are indicated above the sequence. Selected restriction enzyme target sites are identified. efficiency of the promoter regions from the two organisms in an $S$. coelicolor host.

(iii) In $\mathrm{J} 171$, the whiB-Stv coding region gave rapid and full complementation when expressed from the P1 plus $\mathrm{P} 2$ promoter region of $S$. coelicolor. This showed that the three conservative amino acid differences do not significantly affect the protein's function in $S$. coelicolor.

(iv) During microscopic observation of aerial mycelium from the various constructions in $\mathrm{C70}$, in which the mutant phenotype was only partially corrected, we observed a single example of a multiple branch point (C70/pIJ2184, Fig. 8b; this triple branch was not a chance apposition of separate fragments, since it moved as one piece when nearby air bubbles coalesced during microscopic examination). We have never previously seen such branch points in $S$. coelicolor or its whi mutants, but they are typical of Streptoverticillium spp. (Fig. 4).

The whiG genes of Stv. griseocarneum and S. coelicolor are highly similar

The part of the $2.3 \mathrm{~kb}$ Streptoverticillium DNA fragment of pIJ2177 that hybridized to the whiG probe was located, by the exonuclease III method described in the
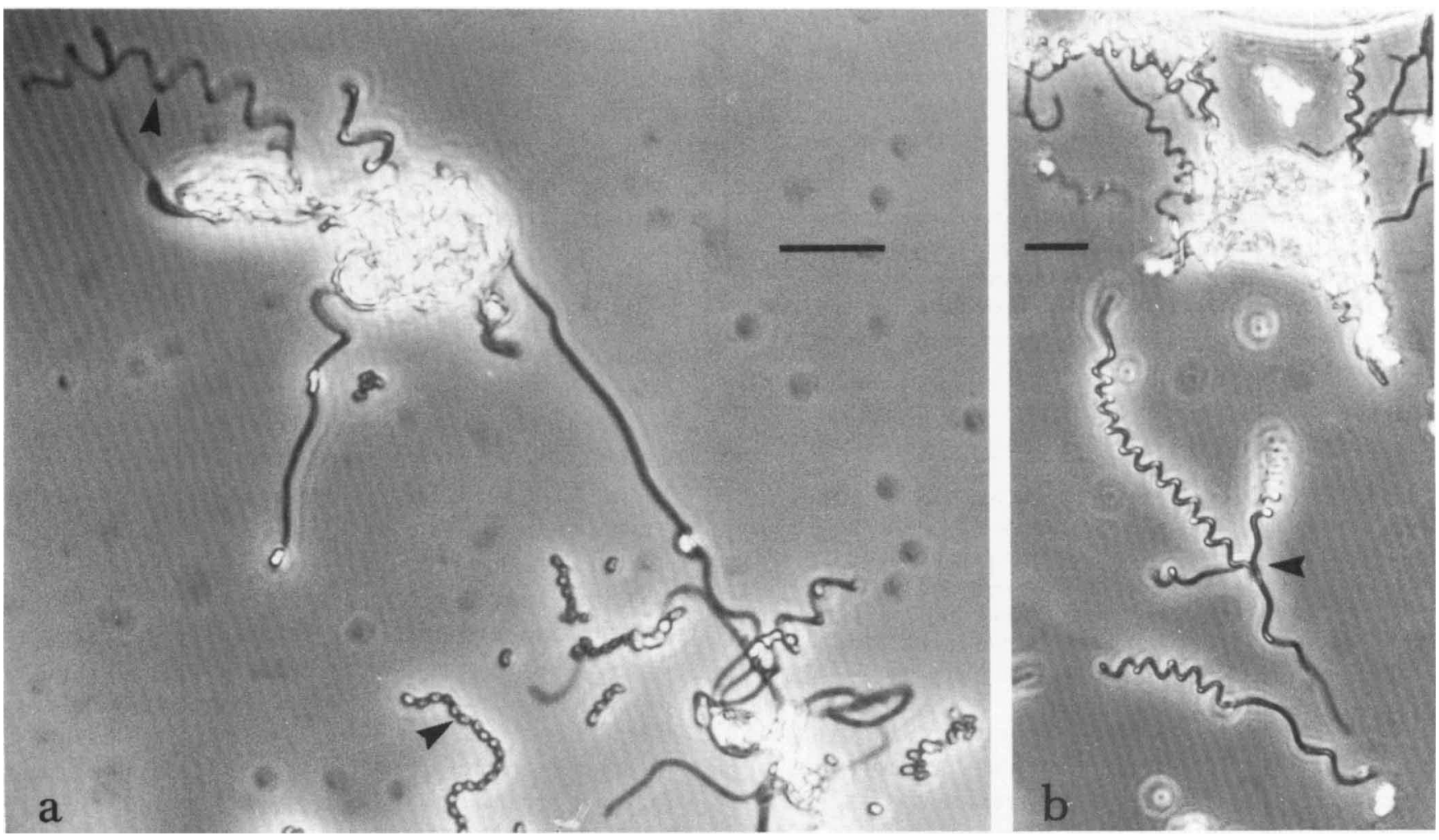

Fig. 8. Phase-contrast micrographs of aerial mycelium. (a) Typical partial complementation of $\mathrm{C} 70$ (S. coelicolor, whiB70), in this case by a plasmid carrying the $S$. coelicolor whiB gene with an $N d e I$ site introduced at the start of its coding sequence, and its natural promoter region. Arrows indicate a spore chain (the wild-type phenotype) and a coiled aerial hypha (the whiB phenotype). (b) A rare three-way branch in an aerial hypha of $\mathrm{C} 70$ (whiB70) bearing a plasmid containing the $S$. coelicolor whiB gene and Pl promoter, but with the P2 promoter region replaced by homologous DNA from Stv. griseocarneum. Bar markers, $10 \mu \mathrm{m}$. 


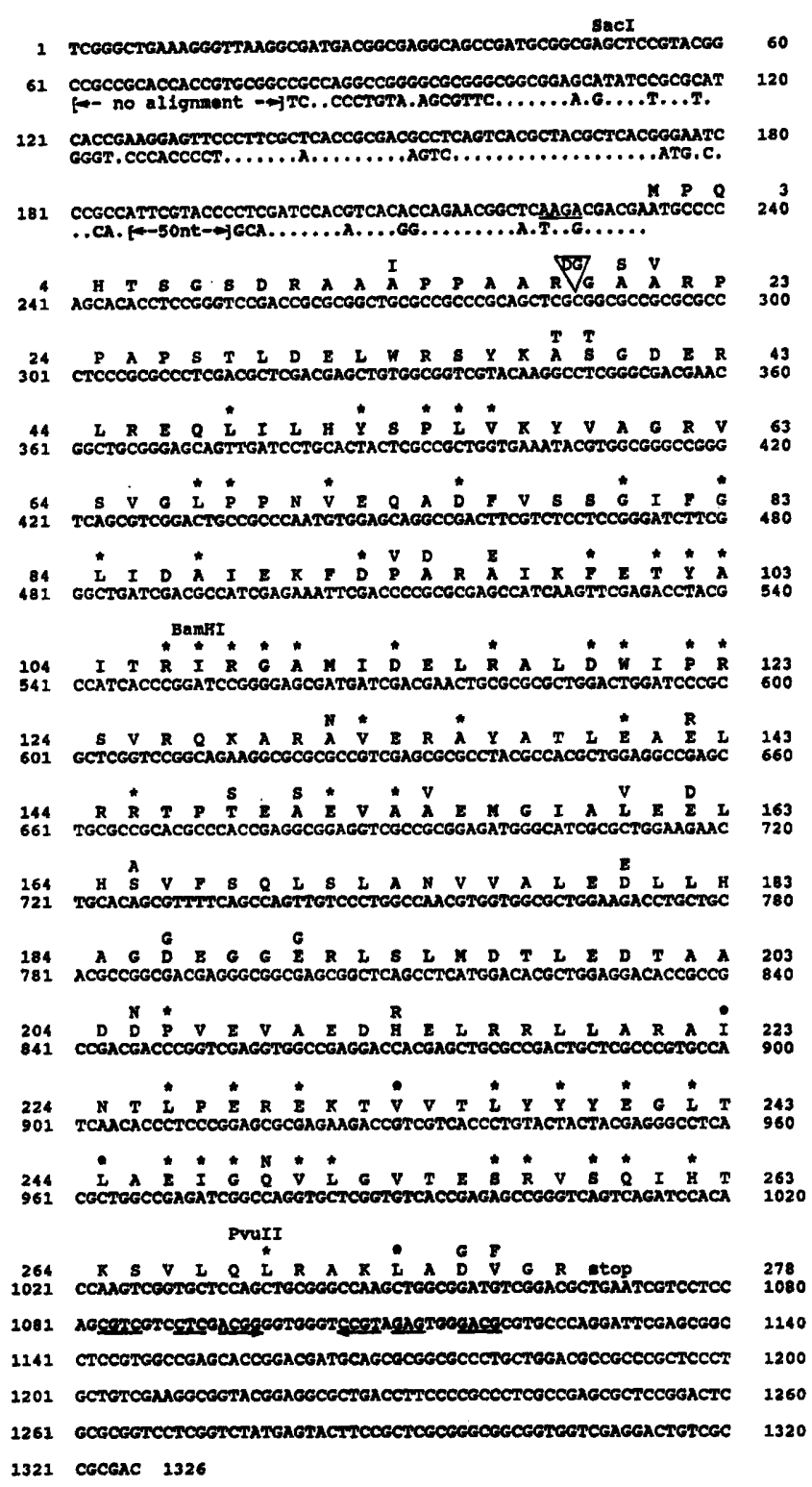

Fig. 9. DNA sequence of whiG-Stv. Symbols are as in Fig. 7, except that comparisons are with the $S$. coelicolor whiG sequence (Chater et al., 1989). Two additional residues present in the $S$. coelicolor whiG gene product are shown in a triangle. A single base gap in the aligned $S$. coelicolor sequence is shown opposite position 120 . In addition, amino acid residues that are marked with asterisks are identical in $\sigma^{D}$ of $B$. subtilis (Helmann et al., 1988), $\sigma^{F}$ of Salmonella typhimurium (Ohnishi et al., 1990) and $\sigma^{\mathrm{F}}$ of Pseudomonas aeruginosa (Starnbach \& Lory, 1992). An imperfect inverted repeat appropriately positioned to act as a terminator is indicated by punctuated horizontal arrows.

preceding section, to a position about 1.3 to $1.6 \mathrm{~kb}$ from the universal-primer-proximal end of the insert (Fig. 5). The sequence of $1326 \mathrm{bp}$ from this region was determined (Fig. 9). Computer analysis revealed a single ORF (termed whiG-Stv) preceded by a weak ribosome-binding site. The deduced protein product of the ORF is closely similar to the $S$. coelicolor whiG gene product $(90 \%$ identity with one gap; at the DNA sequence level, there is $85.4 \%$ identity). Apparent initiation and termination codons are found in the same positions in the two whiG genes. The comparison helps to resolve an uncertainty about the choice of probable translation start site in $S$. coelicolor, in which a second potential start site for whiG was suggested involving the GTG codon corresponding to valine 23 (Chater et al., 1989): a GCG (alanine) codon occupies the corresponding position (21) in whiG-Stv (Fig. 9). It is therefore very likely that the ATG codon indicated in Fig. 9 corresponds to the correct start codon for the $S$. coelicolor whiG gene. None of the sequenced 233 bp upstream of the whiG-Stv ORF showed obvious coding characteristics. Alignment of this region with that upstream of the $S$. coelicolor whiG ORF showed regions of similarity separated by regions of divergence. Notably, the $35 \mathrm{bp}$ immediately upstream of the ORFs differed at only six positions, and another comparably similar segment (five differences in $40 \mathrm{bp}$ ) was observed slightly further upstream (Fig. 9).

\section{Discussion}

Phylogenetic and evolutionary significance of the distribution of whiB-and whiG-related sequences

In all the actinomycete genera represented in this study, a form of fragmentation of rod-shaped or filamentous cells occurs that might be evolutionarily equivalent to sporulation in S. coelicolor, and which might therefore involve genes homologous with whiB and whiG. We found apparent whiB homologues in all the strains, whereas whiG homologues were of more limited occurrence. For one strain, Stv. griseocarneum, the genes were shown to function in $S$. coelicolor. We assume that the hybridizing sequences detected in the other strains are also $w h i B$ or whiG homologues and are functional, though it is not ruled out that some of them may be cryptic or their hybridization entirely fortuitous.

The whiG probe included the DNA specifying the region 2.4 of $\sigma^{\mathrm{WhiG}}$, believed from studies of other $\sigma$ factors to contact the -10 region of cognate promoters (reviewed by Lonetto et al., 1992). The absence from several actinomycetes of DNA similar to the whiG probe therefore indicates a probable absence of $\sigma^{\text {whic }}$-like proteins and of the promoters that would be recognised by a $\sigma^{\text {WhiG }}$ form of RNA polymerase holoenzyme: that is, the probable absence of a $\sigma^{\text {WhiG }}$ regulon. Alternatively, there may be relatively high amino acid sequence divergence among $\sigma^{\text {WhiG }}$ homologues: an interpretation that we do not favour, since $\sigma^{\text {WhiG }}$ proteins from $S$. coelicolor or Stv. griseocarneum are rather similar to homologues from such distant organisms as $B$. subtilis, Salm. typhimurium and $P$. aeruginosa (Helmann, 1991); and since a hybridization signal was obtained with $E$. coli DNA. The organisms in which whiG-related sequences 
were detected differ from those giving no signal, in that they represent genera which can form a sporulating aerial mycelium (Fig. 1). (Nocardia corallina is probably more correctly classified as a Rhodococcus, a genus that typically does not form aerial mycelium: Lechevalier, 1989.) It will be interesting in future to learn whether molecular phylogeny shows $A$. mediterranea and Sac. erythraea to be close to Streptomyces and Streptoverticillium, since the proposed role of whiG in the switch from indeterminate aerial growth to sporulation has potentially high adaptive, and therefore evolutionary, significance. In fact, both organisms were originally described as Streptomyces spp. (Margalith \& Beretta, 1960; Labeda, 1987). On the parsimonious hypothesis that sporulation septation in $S$. coelicolor and fragmentation in other actinomycetes are indeed homologous processes, whiG would appear not to be centrally involved in either process, whereas the omnipresent whiB might play a more direct role. In those organisms from which whiG homologues are absent, transcription of the whiB homologues cannot depend, directly or indirectly, on a $\sigma^{\text {WhiG }}$ form of RNA polymerase. This is consistent with results showing that whiB is transcribed from its normal promoters in a whiG mutant of $S$. coelicolor (Soliveri et al., 1992).

The apparent absence of a whiG homologue from a motile-spored Actinoplanes (even using conditions of reduced stringency: results not shown) was perhaps surprising, because $\sigma^{\text {WhiG }}$ homologues in other Grampositive and Gram-negative bacteria are essential for the transcription of some of the structural genes for flagellum formation (Helmann, 1991). Some flagellar genes of other bacterial groups are transcribed by a form of RNA polymerase holoenzyme containing $\sigma^{54}$ or its homologues (Helmann, 1991), so-among other, more unprecedented, possibilities - such a holoenzyme might conceivably account for the $\sigma^{\mathrm{WhiG}}$-independent expression of flagellum genes in Actinoplanes.

\section{Functional significance of sequence comparisons involving whiB and whiG homologues}

The divergence between $S$. coelicolor and Stv. griseocarneum is greater for whiG than for whiB. The whiB-Stv gene product differs at only three amino acid residues from that of the $S$. coelicolor whiB. At position 5, phenylalanine and valine are alternatives, suggesting a preference for a hydrophobic amino acid at this position; and the glutamate or aspartate alternatives at positions 11 and 12 suggest a requirement for acidic residues in these positions. Davis \& Chater (1992) previously proposed that positions 11 and 12 contributed to the charged face of an amphipathic $\alpha$-helix. The restoration of abundant sporulation to a whiB deletion mutant of $S$. coelicolor by certain plasmids carrying the whiB-Stv coding region shows that these amino acid changes do not significantly impair function in the heterologous genetic background. The whiB-Stv promoter region appears to be somewhat inefficient in $S$. coelicolor, so it is possible that a relatively low level of whiB-Stv transcription may also be the natural situation in its proper host. On the basis of a single observation of a verticil-like structure in a poorly complemented whiB mutant of $S$. coelicolor (Fig. 8), it might even be proposed that the characteristic morphology of Streptoverticillium spp. results from a low level of whiB expression.

The comparison between the two WhiG proteins reveals more divergence than the WhiB comparison, with 28 differences in 279 positions ( $90 \%$ identity, compared with $97 \%$ identity for the WhiB comparison). When the two WhiG proteins are compared with $\sigma^{\mathrm{D}}$ (B. subtilis) and $\sigma^{\mathrm{F}}$ (Salm. typhimurium and $P$. aeruginosa), it emerges that, of the 68 positions identical between $\sigma^{\mathrm{D}}$ and the two $\sigma^{\mathrm{F}}$ sequences, no fewer than $57(84 \%)$ are also identical in the whiG products (asterisks in Fig. 9), suggesting that these are functionally highly significant residues. At a further 90 positions, the two whiG products are identical to each other and to one of the other three $\sigma$ factors. The differences of the whiG-Stv gene product from that of $S$. coelicolor do not seem to affect its ability to function in $S$. coelicolor, as judged by the ability of whiG-Stv DNA at high copy number to cause ectopic sporulation in $S$. coelicolor. We cannot readily explain why this phenotype is not seen when whiG-Stv is introduced into a whiG mutant of $S$. coelicolor.

\section{Concluding remarks}

Clearly, the comparative study of these developmental genes among actinomycetes can give information relevant to their function, regulation and evolution. Further analysis may require disruption of whiB and whiG homologues - a technique currently possible in only a few actinomycetes. These genetically manipulable actinomycetes include members of the genus Mycobacterium. The representative strain that we studied $(M$. tuberculosis var. bovinus BCG) contains a homologue of $w h i B$, but not one of $w h i G$. If the hypothesis that whiB could control fragmentation of these organisms is correct, interference with WhiB function might seriously inhibit their growth, and WhiB could be an interesting target for treatments aimed at mycobacterial infections (especially if whiB homologues are indeed absent from most nonactinomycete bacteria). Besides leprosy, tuberculosis, and probably Crohn's disease and Johne's disease, these infections include opportunistic infections of AIDS sufferers. 
We thank Mervyn Bibb, Mark Buttner, David Hopwood and Tobias Kieser for thoughtful comments on the manuscript; Celia Bruton for help with DNA sequencing; and Meredyth Limberg and Anne Williams for patiently typing successive versions of the manuscript. J.S. and E.V. were supported by Senior Research Fellowships from the European Community, and C.G. by a studentship from the Italian Ministero Pubblica Istruzione. The work was also funded by the John Innes Foundation and the Agricultural and Food Research Council.

\section{References}

BibB, M. J., Findlay, P. R. \& Johnson, M. W. (1984). The relationship between base composition and codon usage in bacterial genes and its use for the simple and reliable identification of protein-coding sequences. Gene 30, 157-166.

Blanco, G., Brian, P., Pereda, A., Méndez, C., Salas, J. A. \& CHATER, K. F. (1993). Hybridization and DNA sequence analyses suggest an early evolutionary divergence of related biosynthetic gene sets for polyketide antibiotics and spore pigments in Streptomyces spp. Gene 130, 107-116.

CHATER, K. F. (1972). A morphological and genetic mapping study of white colony mutants of Streptomyces coelicolor. Journal of General Microbiology 72, 9-28.

CHATER, K. F. (1989). Multilevel regulation of Streptomyces differentiation. Trends in Genetics 5, 372-376.

Chater, K. F., Bruton, C. J., King, A. A. \& SuÁrez, J. E. (1982). The expression of Streptomyces and Escherichia coli drug resistance determinants cloned into the Streptomyces phage $\phi \mathrm{C} 31$. Gene 19, 21-23.

Chater, K. F., Bruton, C. J., Plaskitt, K. A., Butther, M. J., MÉndez, C. \& HelmanN, J. D. (1989). The developmental fate of $S$. coelicolor hyphae depends upon a gene product homologous with the motility $\sigma$ factor of $B$. subtilis. Cell 59, 133-143.

Cross, T., ATTwell, R. W. \& LoCCI, R. (1973). Fine structure of the spore sheath in Streptoverticillium species. Journal of General Microbiology 75, 421-424.

DAvIs, N. K. \& ChATER, K. F. (1992). The Streptomyces coelicolor whiB gene encodes a small transcription factor-like protein dispensable for growth but essential for sporulation. Molecular and General Genetics 232, 351-358.

DevereuX, J., Haeberli, P. \& Smithies, O. (1984). A comprehensive set of sequence analysis programs for the VAX. Nucleic Acids Research 12, 387-395.

DownIE, J. A. \& SuRIN, B. P. (1990). Either of two nod gene loci can complement the nodulation defect of a nod deletion mutant of Rhizobium leguminosarum bv. viciae. Molecular and General Genetics 222, 81-86.

Goodfellow, M. (1989). Suprageneric classification of the actinomycetes. In Bergey's Manual of Systematic Bacteriology, vol. 4, pp. 2333-2339. Edited by S. T. Williams, M. E. Sharpe \& J. G. Holt. Baltimore: Williams \& Wilkins.

Goodfellow, M. \& Cross, T. (1983). Classification. In The Biology of the Actinomycetes. pp. 7-164. Edited by M. Goodfellow, M. Mordarski \& S. T. Williams. London: Academic Press.

HelmanN, J. D. (1991). Alternative sigma factors and the control of flagellar gene expression. Molecular Microbiology 5, 2875-2882.

Helmann, J. D., Marquez, L. M. \& Chamberlin, M. J. (1988). Cloning, sequencing and disruption of the Bacillus subtilis $\sigma^{28}$ gene. Journal of Bacteriology 170, 1568-1574.

HENIKOFF, S. (1984). Unidirectional digestion with exonuclease III creates targeted breakpoints for DNA sequencing. Gene 28, 351-359.

Hopwood, D. A., Wildermuth, H. \& PALMER, H. M. (1970). Mutants of Streptomyces coelicolor defective in sporulation. Journal of General Microbiology 61, 397-408.

Hopwood, D. A., Bibb, M. J., Chater, K. F., Kieser, T., Bruton,
C. J., Kieser, H. M., Lydiate, D. J., Smith, C. P., WARD, J. M. \& SCHREMPF, H. (1985). Genetic Manipulation of Streptomyces. A Laboratory Manual. Norwich: John Innes Foundation.

KUNKEL, T. A., RoBerTs, J. D. \& ZAKOUR, R. A. (1987). Rapid and efficient site-directed mutagenesis without phenotypic selection. Methods in Enzymology 154, 367-382.

LABEDA, D. P. (1987). Transfer of the type strain of Streptomyces erythreus (Waksman 1923) Waksman and Henrici 1948 to the genus Saccharopolyspora Lacey and Goodfellow 1975 as Saccharopolyspora erythraea sp. nov. International Journal of Systematic Bacteriology 37, 19-22.

LECHEVALIER, H. A. (1989). Nocardioform actinomycetes. In Bergey's Manual of Systematic Bacteriology, vol. 4. pp. 2348-2404. Edited by S. T. Williams, M. E. Sharpe \& J. G. Holt. Baltimore: Williams \& Wilkins.

LocCI, R. (1989). Streptomyces and related genera. In Bergey's Manual of Systematic Bacteriology, vol. 4. pp. 2451-2508. Edited by S. T. Williams, M. E. Sharpe \& J. G. Holt. Baltimore: Williams \& Wilkins.

Locci, R., Baldacci, E. \& Petrolini-Baldan, B. (1969). The genus Streptoverticillium. A taxonomic study. Giornale di Microbiologia 17, $1-60$.

Lonetro, M., Gribskov, M. \& Gross, C. A. (1992). The $\sigma^{70}$ family: sequence conservation and evolutionary relationships. Journal of Bacteriology 174, 3843-3849.

Margalith, P. \& Beretta, G. (1960). Rifamycin. XI. Taxonomic study on Streptomyces mediterranei nov. sp. Mycopathologia et Mycologia Applicata 13, 321-330.

McVITTIE, A. M. (1974). Ultrastructural studies on sporulation in wild-type and white colony mutants of Streptomyces coelicolor. Journal of General Microbiology 81, 291-302.

MÉnDEZ, C. \& CHATER, K. F. (1987). Cloning of whiG, a gene critical for sporulation of Streptomyces coelicolor A3(2). Journal of Bacteriology 169, 5715-5720.

Norrander, J., Kempe, T. \& Messing, J. (1983). Construction of improved M13 vectors using oligonucleotide-directed mutagenesis. Gene 26, 101-106.

Ohnishi, K., Kutsukake, K., Suzuki, H. \& InNo, T. (1990). Gene fliA enclodes an alternative sigma factor specific for flagella operons in Salmonella typhimurium. Molecular and General Genetics 221, 139-147.

Sambrook, J., Fritsch, E. F. \& Maniatis, T. (1989). Molecular Cloning: a Laboratory Manual, 2nd edn. Cold Spring Harbor, NY: Cold Spring Harbor Laboratory.

SANGER, F., Nicklen, S. \& Coulson, A. R. (1977). DNA sequencing with chain-terminating inhibitors. Proceedings of the National Academy of Sciences of the United States of America 74, 5463-5467.

Soliveri, J., Brown, K. L., ButtNer, M. J. \& Chater, K. F. (1992). Two promoters for the whiB sporulation gene of Streptomyces coelicolor $\mathrm{A} 3(2)$, and their activities in relation to development. Journal of Bacteriology 174, 6215-6220.

Starnbach, M. N. \& LoRY, S. (1992). The flia (rpoF) gene of Pseudomonas aeruginosa encodes an alternative sigma factor required for flagellin synthesis. Molecular Microbiology 6, 459-469.

TAN, H. \& CHATER, K. F. (1993). Two developmentally controlled promoters of Streptomyces coelicolor A3(2) that resemble the major class of motility-related promoters in other bacteria. Journal of Bacteriology 175, 933-940.

WARD, J. M., JANSSEN, G. R., Kieser, T. \& BibB, M. J. (1986). Construction and characterisation of a series of multi-copy promoter-probe plasmid vectors for Streptomyces using the aminoglycoside phosphotransferase gene from $\mathrm{Tn} 5$ as indicator. Molecular and General Genetics 203, 468-478.

Wildermuth, H. \& HopwoOd, D. A. (1970). Septation during sporulation in Streptomyces coelicolor. Journal of General Microbiology 60, 51-59.

Yanisch-PerRon, C., Vieira, J. \& Messing, J. (1985). Improved M13 vectors and host strains: nucleotide sequences of the M13mp18 and pUC19 vectors. Gene 33, 103-119. 